\title{
Uterine perforation by GyneFix frameless IUD: Two case reports
}

Jagdish D Gandhi, MD, FRCOG, MFFP, Specialist Registrar, Liverpool Women's Hospital; Jane Whitmore, MBBS, MFFP, MIPM, Consultant, Family Planning and Reproductive Health, North Sefton and West Lancs Community NHS Trust; Mohsen N Iskander, FRCOG, Consultant and Head of Department, Obstetrics and Gynaecology Department, Southport and Ormskirk Hospitals.

Correspondence: Mr J.D. Gandhi, Department of Gynaecology, Southport District General Hospital, Towns Lane, Kew, Southport PR8 6NJ, UK. Tel/Fax: 01695625900

(Accepted $18^{\text {th }}$ December, 2000)

The Journal of Family Planning and Reproductive Health Care 2001: 27(3): 153-154

\begin{abstract}
Two cases of uterine perforation are described, occurring 11 days and 4 months, respectively, after the insertion of GyneFix, a frameless intra-uterine contraceptive device (IUD). In both the cases initial ultrasound scan showed the intra-uterine position of the device. Removal of the IUD, either by laparoscopy or laparotomy, had to be carried out. Awareness of this complication, insertion of GyneFix by a trained operator, appropriateness of ultrasound scan monitoring and possible underreporting of this complication are discussed.
\end{abstract}

\section{Key message points \\ - Perforation of the uterus can occur with the frameless IUD GyneFix. - Skilful insertion by a trained operator is essential. \\ - Use of ultrasound scanning in problem cases requires close collaboration with ultrasonographers. \\ - Reporting all perforations by GyneFix and evidence-based information is advocated.}

\section{Case 1}

A 28-year-old nulliparous lady requested the insertion of an IUD, and a community family planning consultant subsequently inserted a GyneFix. A normal pelvic examination was noted and the GyneFix insertion was uneventful. At the time of the clinic attendance, she had been amenorrheic for 2.5 years on the progestogen-only pill, and was in a stable relationship. Eighteen months prior to this consultation she was seen in gynaecological clinic because of dyspareunia, and was suspected to have chronic pelvic inflammatory disease. Her Chlamydia culture was positive, with a co-existent Gardnerella infection for which she received doxycycline and Flagyl. At the time of GyneFix insertion she was entirely asymptomatic, hence further Chlamydia tests were not performed.

Two days after insertion of GyneFix, she presented to her general practitioner (GP) complaining of chest pain and lower abdominal pain. She was seen at the local hospital in the gynaecology department where a clinical examination was unremarkable. An ultrasound scan examination showed an IUD in situ. She was reassured. However, in view of her prolonged amenorrhoea, a serum B-HCG was performed and was found to be less than 2 units per litre (normal range $0-10 \mathrm{u} / \mathrm{L}$ ). It was planned that she should be reviewed in 4 weeks' time in the gynaecological clinic.

One week following insertion of GyneFix she reattended the family planning clinic with crampy lower abdominal pain and requested removal of the GyneFix IUD. Clinical examination failed to locate the thread of the device, but transvaginal scan showed the IUD in situ. An attempt was made to remove the IUD under general anaesthetic, after dilatation of the cervix and using polyp removal forceps. However no device was felt in the cavity. On uterine sounding a small perforation was inadvertently made in the posterior uterine wall near the fundus. She was observed on the gynaecological ward and was commenced on prophylactic antibiotics in view of perforation of the uterus.

In the next 48 hours her abdominal pain continued and she had ultrasound scan and an X-ray of her abdomen. The ultrasound scanning failed to show the IUD in the uterus, and X-ray revealed it to be in the pelvis.

The patient underwent laparoscopic removal of the IUD 11 days after insertion. Laparoscopy was performed using the standard technique and carbon dioxide for insufflation. A normal sized uterus, tubes and ovaries with no signs of infection were noted. Approximately $20 \mathrm{mls}$ of bloodstained fluid in the pouch of Douglas was noted, with a 5-7 $\mathrm{mm}$ sized uterine perforation on the right side of the fundus. There was no bleeding from this site and the perforation appeared to be a week old.

The abdominal cavity was explored with the laparoscope and the GyneFix IUD was found in the layers of the small intestine. The knot end of the GyneFix was embedded in the layers of the small bowel, but was able to be detached with a gentle pull using atraumatic-grasping forceps. It was removed laparoscopically. The patient was allowed home 24 hours post-operatively as her symptoms had settled.

She was reviewed in the gynaecological clinic 4 weeks after laparoscopic removal of the IUD. She complained of lower abdominal pain. Clinical examination as well as transvaginal scanning of pelvic organs was unremarkable. She was reassured and was reviewed 4 weeks later. Her abdominal pain settled and she was discharged to her GP.

\section{Case 2}

A 34-year-old single nursery teacher attended the community family planning clinic requesting IUD insertion. She had undergone a surgical termination of pregnancy 18 years previously, and had had no other pregnancies.

A consultant in the family planning services fitted a GyneFix IUD in late March 1999. The procedure was performed by the standard technique and was uneventful. She re-visited the clinic after 1 week, when the thread was not visible. However, an ultrasound scan confirmed the IUD in the uterus.

Approximately 3 months after having the IUD fitted, she contacted her GP because of a heavy vaginal bleed with 
clots, and feeling weak and tired. Her bleeding settled on a course of tranexamic acid. However, clinical examination 2 weeks later showed a bulky uterus and a pregnancy test was positive. She was referred to the local hospital for further assessment.

Approximately 14 weeks after the IUD insertion when she attended the hospital, she reported that her last period was delayed by 2 days. Although her general condition was satisfactory, her haemoglobin was $6.9 \mathrm{~g} / \mathrm{dl}$. An ultrasound scan examination showed a $22 \mathrm{~mm}$ irregular gestation sac equivalent to 7 weeks 6 days by gestation age. A foetal echo was seen, but there was no foetal heart activity. The gestation sac was cloudy. The IUD was not seen in the uterus. X-ray examination showed the IUD present in the lower abdomen. A clinical diagnosis of missed abortion with lost IUD in the abdomen was established.

The patient received 4 units of blood transfusion and underwent suction evacuation of the uterus, laparoscopy and laparotomy. Laparoscopic examination showed the IUD thread to be buried in the omentum. A gentle tug during the laparoscopic procedure could not retrieve the IUD. She underwent a laparotomy where the lost GyneFix IUD was recovered, buried in inflammatory omentum, which was also dissected and sent for histological examination. This confirmed intense acute and chronic inflammation amounting to chronic abscess formation with associated necrosis in the omental tissue. Material from evacuation of the uterus confirmed unremarkable first trimester chorionic villi.

The patient made an uneventful recovery and was reviewed 6 weeks post-operatively in the gynaecology clinic, when she was discharged.

\section{Discussion:}

Perforation of the uterus is one of the most serious complications associated with insertion of an IUD. The frequency has been estimated between 0.05 and 13 per 1000 insertions, varying according to a number of factors including the device used and the operator's experience. ${ }^{1,2}$

The frameless intra-uterine implant system (GyneFix) was conceived in 1985 and was welcomed as a very useful addition to the contraceptive menu. GyneFix consists of six copper sleeves, each $5 \mathrm{~mm}$ long and $2.2 \mathrm{~mm}$ in diameter, threaded on a length of suture material. The proximal end of the thread is provided with a knot, which is placed in the fundal myometrium with an inserter, at a controlled depth of $1.0 \mathrm{~cm}$, and acts as an anchoring system. The insertion technique is different to all other IUDs and in the UK all doctors who wish to fit GyneFix have to undergo special training. The insertion technique has been described previously. ${ }^{3}$

Perforation at insertion, delayed perforation, or migration has not been recorded in large international multicenter clinical trials with the frameless device. ${ }^{4,5}$ It might be supposed that a frameless device anchored in the myometrium might erode through more easily than a framed device. The absence in perforation rates may refute this supposition although there may be considerable underreporting. More recently a recent product surveillance report from Belgium on the first 15000 insertions revealed a perforation rate of $1.4 / 1000$ in the first year, to $1 / 1000$ in the third year.

Uterine perforation by an IUD may depend on the operator's experience, design of the device or thinness of the uterine wall. In order to obtain optimal results, every IUD needs skilful insertion. In the cases we report an experienced doctor performed the GyneFix insertion and the procedures were uneventful. Both patients, however, sought medical advice within the first week of the insertion and hence perforation at the time of insertion cannot be ruled out.

These two case reports emphasise the need for clinicians and users to be aware that perforation of the uterus can occur with GyneFix. This is of particular relevance to GPs, who may not be fitting GyneFix themselves, but may be the first port of call for users when problems arise. GPs need to be aware both of the management of lost threads and other problems that occur in IUD users, and of the differences between GyneFix and framed devices.

Although in a long-term ultrasound scan follow-up study $^{6}$ of 405 women who used GyneFix for 5 years there was absence of migration of anchor, these cases raise the possibility of deep embedding of the knot and subsequent migration of the device.

The first case raises the question whether prolonged amenorrhoea whilst on the progestogen-only pill leads to a thin myometrium, making the uterus more prone to perforation. However, this is purely a conjectural issue. This case made us question whether it would be appropriate to measure myometrial thickness routinely, by ultrasound scan, either before insertion or at the follow-up. Although such a policy might identify those IUD users most at risk of perforation, in practice this is not a feasible proposition in our service. However, if difficulty is experienced at the time of insertion, or if the patient develops abdominal pains, irregular vaginal bleeding, temperature or lost threads, then ultrasonography may be helpful.

The first case demonstrates how important it is that ultrasonographers are aware of the difference between framed and non-framed devices and the importance of the position of the first copper bead and the thickness of the fundal myometrium; otherwise misleading scan results might result in inappropriate management. It has been recommended to remove the GyneFix if the distance between the peritoneal surface of the uterus and the first copper sleeve is less than $9 \mathrm{~mm}$ or greater than $20 \mathrm{~mm}^{6}$

Finally, all known cases of IUD perforations, at insertion and delayed, should be reported to the Medical Devices Agency. This is particularly important in the case of GyneFix, given its frameless design and intra-myometrial anchoring device. GyneFix is a highly effective, welltolerated intra-uterine device for nulliparous and multiparous women. It would be reassuring to have robust evidence-based information that perforations, both immediate and delayed, are no more common than with other IUDs.

\section{Acknowledgements}

The authors wish to thank Mr Sanjeev Sharma FRCOG, District Tutor, RCOG, Southport District General Hospital and Miss Anne Garden MD FRCOG, Senior lecturer in Obstetrics and Gynaecology at Liverpool University for their help preparing these case reports.

\section{Statements on funding and competing interests \\ Funding: None \\ Competing interests: None}

References

Oborne JL, Bennett MJ. Removal of intra-abdominal intrauterine contraceptive devices. $\mathrm{Br}$ Obstet Gynaecol 1978: 85:868-871.

Søgaard K. Unrecognised perforation of the uterine and rectal walls by an intrauterine contraceptive device. Acta Obstet Gynaecol Scand 1993; 72: 55-56.

Wildemeersch D, Batar I, Gholade BA, et al. Gynefix: The intra-uterine contraceptive implan - an update for interval, emergency and interval contraception. The British Journal of Family Planning 1999; 24: 149-159.

UNDP, UNFPA and WHO Special Programme for Research Development and Research Training in Human Reproduction, World Bank: IUD Research Group. The $\mathrm{Cu}$ 380A IUD and the Frameless "Flexi Gard". Interim Three-Year Data from an international multicenter Trial. Contraception 1995;52:77-83.

Van Kets H, Vrijens M, Van Trappen y, et al. The Gynefix intrauterine implant: a majo Improvement in efficacy, expulsion and tolerance. Adv Contracept 1995; 11: 131-142.

6 Wildemeersch D, Defoori P, Martens G, The Flexigard 330ICC, an ultrasound evaluation. Contraception 1992; 47: 471-476. 